\title{
Finite-size domains in membranes with active two-state inclusions
}

\author{
Chien-Hsun Chen ${ }^{(1),(2)}$ and Hsuan-Yi Chen ${ }^{(1),(2),(3)}$ \\ Department of Physics(1), \\ Center for Complex Systems ${ }^{(2)}$, \\ and Graduate Institute of Biophysics ${ }^{(3)}$, \\ National Central University, \\ Jhongli, 32054 \\ Taiwan
}

(Dated: October 30, 2018)

\begin{abstract}
The distribution of inclusion-rich domains in membranes with active two-state inclusions is studied by simulations. Our study shows that typical size of inclusion-rich domains $(L)$ can be controlled by inclusion activities in several ways. When there is effective attraction between state1 inclusions, we find: (i) Small domains with only several inclusions are observed for inclusions with time scales $\left(\sim 10^{-3} \mathrm{~s}\right)$ and interaction energy $\left[\sim \mathcal{O}\left(\mathrm{k}_{\mathrm{B}} \mathrm{T}\right)\right]$ comparable to motor proteins. (ii) $L$ scales as $1 / 3$ power of the lifetime of state- 1 for a wide range of parameters. (iii) $L$ shows a switch-like dependence on state-2 lifetime $k_{12}^{-1}$. That is, $L$ depends weakly on $k_{12}$ when $k_{12}<k_{12}^{*}$ but increases rapidly with $k_{12}$ when $k_{12}>k_{12}^{*}$, the crossover $k_{12}^{*}$ occurs when the diffusion length of a typical state-2 inclusion within its lifetime is comparable to $L$. (iv) Inclusion-curvature coupling provides another length scale that competes with the effects of transition rates.

PACS numbers: 87.16.Dg, 05.40.-a, 05.70.Np
\end{abstract}




\section{INTRODUCTION}

Biological membranes are composed of lipids, proteins, carbohydrates and other materi-

als [1]. Many experimental as well as theoretical studies have shown that the distribution of these molecules in a membrane is nonuniform. Instead, dynamical patch-like structures with typical size ranging from tens of nanometers to about one micrometer exist in a membrane [2]. The mechanism for the formation of these heterogeneous structures has been a major research topic in membrane biophysics in recent years. Possible mechanisms include (i) continuous membrane recycling may result in nonequilibrium domains in biological membranes [3], and (ii) the coupling between lipid density and local membrane curvature provides a length scale for membrane domains [4]. The most important conclusion from these studies is that finite-size domains in membranes have to be sustained either by nonequilibrium processes such as recycling or by effective long-range interactions due to membrane elasticity.

Another important trend in recent biomembrane research is to treat biomembranes as nonequilibrium systems [5, 6, 7, 8, 99, 10, 11, 12, 13] which contain active inclusions that are driven by external stimuli. Earlier work on these active membranes studied mainly the effect of nonequilibrium activities on their fluctuation spectrums [10, 11, 12], the instabilities due to activities were also discussed in [11, 13]. In these models, the effect of conformation change of the inclusions are neglected. On the other hand, it has been shown by Lacoste and Lau [6] that active inclusions with an internal time scale have different fluctuation power spectrum from simpler models which neglects this effect. Furthermore, finite-wavelength instabilities were predicted in [5, 7, 8, 9] for membranes with two-state active inclusions, and it has been pointed out in [5] that these instabilities are similar to the dynamic nm-scale domains in biomembranes. Indeed, recent experiments have observed enhanced spatial aggregation of bacteriorhodopsin (BR) due to light-induced activities in model membranes [14] and clustering of light-harvesting antenna domains in photosynthetic membranes under lowlight conditions [15]. These experimental observations suggest that size of inclusion-rich domains in a membrane can be tuned by changing the strength of external stimuli of the active inclusions. Therefore in this article we study the physics of activity-controlled finite size domains in membranes in a simple model.

To focus on the basic interactions responsible for inclusion-activity induced finite size membrane domains, we consider the simplest possible model, i.e., a fluid membrane com- 
posed of one type of lipid and two-state active inclusions. An inclusion is an active unit which corresponds to a molecular complex that contains proteins, lipids, and other molecules. As shown schematically in Fig. 1, inclusions in different internal states have different conformations, therefore the interactions between the inclusions and lipids depend on the internal states of the inclusions. There are also couplings between the inclusion densities and membrane curvature due to the up-down shape asymmetry of the inclusions. Lattice Monte Carlo simulations are applied to study the steady-state distribution of the inclusions when there is attractive interaction between state-1 inclusions. The effect of inclusion-curvature couplings and inclusion transition rates $k_{\alpha \beta}$ (probability that a state- $\beta$ inclusion transforms to state $\alpha$ per unit time) on the typical size of inclusion-rich domains is discussed. The main results of our simulations are: (i) Small domains with only several inclusions are observed for inclusions with time scales $\left(\sim 10^{-3} \mathrm{~s}\right)$ and interaction energy $\left[\sim \mathcal{O}\left(\mathrm{k}_{\mathrm{B}} \mathrm{T}\right)\right]$ comparable to motor proteins, i.e., our simple model is able to produce the kind of small inclusion clusters observed in [14] within typical parameter range. (ii) Typical size of inclusion-rich domain (L) scales as $L \sim k_{21}^{-1 / 3}$. This often observed behavior [3, 7, 8] provides a mechanism to control continuously the size of inclusion-rich domains by inclusion activities. (iii) A switch-like response of typical size of inclusion-rich domains to the external stimuli is found at fixed $k_{21}$ : There exists a crossover transition rate $k_{12}^{*}$ such that $L$ depends weakly on the stimuli $\left(k_{12}\right)$ when $k_{12} \lesssim k_{12}^{*}$ but becomes very sensitive to it (i.e., $L$ increases rapidly as $k_{12}$ increases) when $k_{12} \gtrsim k_{12}^{*}$. The crossover $k_{12}^{*}$ occurs when the diffusion length of a state-2 inclusion within its lifetime is about the same as $L$. (iv) $L$ decreases when the coupling between state-1 inclusion and membrane curvature increases.

\section{MODEL}

The system is described by a lattice model with membrane height $h(i, j)$, inclusion densities $\phi_{\alpha}(i, j)(\alpha=1$ or 2$)$, and lipid density $\phi_{0}(i, j)$ defined on a two-dimensional square lattice. The Hamiltonian of the system $H=H_{m}+H_{i}+H_{c}$ includes the elastic energy of the membrane, the interaction energy between the inclusions and lipid molecules, and the inclusion-membrane coupling. To lowest order

$$
H_{m}=\frac{1}{2} \sum_{(i, j)} a^{2}\left[\kappa\left(\nabla_{\perp}^{2} h(i, j)\right)^{2}+\gamma\left(\nabla_{\perp} h(i, j)\right)^{2}\right],
$$


where $\kappa$ is the bending rigidity, $\gamma$ is the surface tension of the membrane, $a$ is the lattice constant, $\nabla_{\perp}^{2} h(i, j)=[h(i+1, j)+h(i-1, j)+h(i, j+1)+h(i, j-1)-4 h(i, j)] / a^{2}$, and $\nabla_{\perp} h(i, j)=\hat{\mathbf{i}}[h(i+1, j)-h(i-1, j)] / 2 a+\hat{\mathbf{j}}[h(i, j+1)-h(i, j-1)] / 2 a . \quad H_{i}$ includes the short-range interactions between the inclusions and the lipids, the simplest form is nearestneighbor interaction

$$
H_{i}=\sum_{\langle(i, j)(k, l)\rangle} \sum_{m, n=0}^{2} J_{m n} \phi_{m}(i, j) \phi_{n}(k, l),
$$

where $\sum_{m=0}^{2} \phi_{m}(i, j)=1$ for all $(i, j), \sum_{\langle(i, j)(k, l)\rangle}$ means sum over all nearest-neighboring pairs. The simplest form for the coupling between the inclusion density and local membrane curvature is

$$
H_{c}=\sum_{(i, j)} \sum_{m=0}^{2} \kappa c_{m} \phi_{m}(i, j)\left[\nabla_{\perp}^{2} h(i, j)\right]
$$

where $c_{0}$ is the spontaneous curvature of the lipids, $c_{\alpha}(\alpha=1,2)$ is the coupling between state- $\alpha$ inclusions and local membrane curvature. The inclusion-curvature coupling constant $c_{\alpha} \sim \Delta \Sigma_{\alpha} / \Sigma_{\alpha} l_{\alpha}$ has dimension of inverse length, where $\Sigma_{\alpha}$ is the average cross sectional area of a type- $\alpha$ inclusion, $\Delta \Sigma_{\alpha}$ is the difference between head area and $\Sigma_{\alpha}$, and $l_{\alpha}$ is the thickness (in the $z$ direction) of the inclusion [16].

The simulations are performed on a $L_{M} \times L_{M}$ lattice with periodic boundary condition. The lattice constant $a \sim 5 \mathrm{~nm}$ is chosen to be the smallest length scale for the continuum elasticity theory of membranes to be valid [17]. In the simulations each lattice site is either occupied by a state- 1 inclusion, a state- 2 inclusion, or lipids. That is, $\phi_{m}(i, j)=0$ or $1(m=0,1$, or 2$)$, and $\sum_{m=0}^{2} \phi_{m}(i, j)=1$ for all $(i, j)$. Since our goal is to study the effect of $k_{\alpha \beta}$ and $c_{m}$ on the distribution of the inclusion-rich domains, in the present study the active forces exerted by the inclusions and the viscous flow of the solvent are, similar to another active membrane simulation [18], neglected. Each Monte Carlo step is composed of three independent trial moves: the motion of the membrane, the in-plane motion of the inclusions, and the conformation change of the inclusions. The dynamics of $h(i, j)$ is simulated by updating the membrane height of a randomly chosen lattice site with a displacement between $-\Delta h$ and $\Delta h$ with Metropolis algorithm, where $\Delta h=0.4$ $\mathrm{nm}$. The in-plane motion of the inclusions is simulated by Kawasaki exchange dynamics. Time interval of one Monte Carlo step can be derived from the two-dimensional diffusion 
law $\left\langle r^{2}\right\rangle=4 D t$. Since typical diffusion constant for membrane proteins is on the order of $1 \mu \mathrm{m}^{2} / \mathrm{s}$ [19], and a free inclusion moves a distance $a$ in one Monte Carlo step, a single Monte Carlo step corresponds to time interval $\Delta t \sim 10^{-5}$ s. The inclusion conformation changes are simulated by assigning each state- $\beta$ (state- $\alpha$ ) inclusion a probability $k_{\alpha \beta} \Delta t$ $\left(k_{\beta \alpha} \Delta t\right)$ to change its state to state $\alpha($ state $\beta)$ in each Monte Carlo step. We choose $k_{\alpha \beta}$ 's to be constants in the simulations, i.e., we assume that the coupling between conformation change of the inclusions and local membrane curvature and composition is small. Therefore the conformation change does not obey detailed balance, the steady state of the system is not in thermal equilibrium [20]. In addition, in this article all the simulations are performed with $k_{\alpha \beta} \Delta t<\mathcal{O}(1)$ such that the lifetime of each state is sufficiently long that inclusion diffusion can be taken into account properly. The initial condition of the simulations is randomly distributed $N_{\text {inc }}=\phi_{\text {inc }} \times L_{M} \times L_{M}$ inclusions with $N_{\text {inc }} / 2$ of them in state 1 and $N_{\text {inc }} / 2$ of them in state 2 in a flat membrane. The probability that an arbitrary inclusion is within an inclusion cluster of $M$ sites, $P(M)$, is analyzed when the systems have reached steady states. Typical size of inclusion-rich domain is taken as $L=\sqrt{M_{\max }}$, where $M_{\max }$ is the value of $M$ which maximizes $P(M)$.

The free parameters in our model include $c_{m}, J_{m n}(m=0,1,2)$, and $k_{12}, k_{21} . J_{m n}$ arises from short range noncovalent interactions between the molecules including the effect of hydrophobic length mismatch between different molecules or inclusions of different internal states. Since we are interested in the case where finite-size inclusion-rich domains exist in the steady state, $J_{m n}$ are chosen such that state-1 inclusions attract each other, and state-2 inclusions have very weak or no attraction with other inclusions and lipids. To prevent aggregation of state-2 inclusions due to membrane curvature-induced attraction, $c_{2}$ has to be close to $c_{0}$. For state- 1 inclusions, there is no such restriction on the choice of $c_{1}$. The simplest choice of parameters that satisfies the above criteria is $c_{0}=c_{2}=0$, $c_{1} \geq 0, J_{00}=J_{11}=J_{22}=J_{02}=J$, and $J_{01}=J_{12}=J+\Delta J$ with $\Delta J>0$, and this choice is applied to our simulations. The bending elastic modulus and surface tension of the membrane are chosen to be typical values, $\kappa=5 \times 10^{-20} \mathrm{~N} \cdot \mathrm{m}[21]$, and $\gamma=24 \times 10^{-6}$ $\mathrm{N} \cdot \mathrm{m}[22] . \quad \Delta J \sim \mathcal{O}\left(\mathrm{k}_{\mathrm{B}} \mathrm{T}\right)$ because the interactions between inclusions and lipids are noncovalent, typically $c_{1} \sim \Delta \Sigma_{1} / \Sigma_{1} l_{1} \lesssim \mathcal{O}\left(1 \mathrm{~nm}^{-1}\right)$. For convenience we define a dimensionless constant $G=c_{1} a \sqrt{k_{B} T / \kappa}$ to describe the inclusion-curvature coupling, its typical value is $G \lesssim \mathcal{O}(1)$ 
The transition rates $k_{\alpha \beta}$ for the inclusions depend on the mechanism of conformation transitions of the inclusions under consideration. (i) For inclusions have an intrinsic time scale (like ion pumps), an inclusion stays at state 1 (2) in the absence of stimuli, and changes its conformation to state 2 (1) when it binds to energy source such as ATP or specific ions, the lifetime of state 2 (1) is an intrinsic property of the inclusion, it is on the order of $10^{-2}-10^{-3} \mathrm{~s}$, therefore $k_{12}\left(k_{21}\right) \sim 10^{2}-10^{3} \mathrm{~s}^{-1}$. (ii) For inclusions that change conformation by binding to or dissociating with small ligands, the transition rates $k_{12}, k_{21}$ can exceed $10^{3} \mathrm{~s}^{-1}$, depending on the concentration of ligands in the solvent.

\section{DISCUSSION}

Figure 2 shows domain size distribution $P(M)$ for $\phi_{\text {inc }}=12.5 \%, \Delta J=1.5 k_{B} T$, $k_{12}=10^{2} \mathrm{~s}^{-1}, k_{21}=k_{12} / 32$, and $G=0,1$, and 2 . The peak at $M=1$ comes from the isolated inclusions in the inclusion-poor domain. The peak at greater $M$ provides the characteristic size of inclusion-rich domains. As $G$ increases, the characteristic size of inclusion-rich domains decreases and the peak of $P(M)$ becomes more significant due to inclusion-membrane coupling. This effect can be better visualized by the snap shots from the steady states. As shown in Fig. 3, when $G \neq 0$ the location of inclusion-rich domains has strong correlation with the regions with high membrane curvature. Since the system has lower free energy when the inclusions are in the regions with higher curvature, thus the membrane forms many mountain-like regions with inclusions in them. This effect makes the width of $P(M)$ decrease as $G$ increases and the position of the peak shifts to smaller $M$.

Figure 4 shows the relation between $L$ and $k_{21}$ for $\phi_{\text {inc }}=12.5 \%, \Delta J=1.5 k_{B} T$, and $k_{12}=10^{-2} \mathrm{~s}^{-1}$ on a $128 \times 128$ lattice. The choice of $k_{12}$ is made to study inclusions with an intrinsic time scale $\sim 10 \mathrm{~ms}$, or inclusions in a solution with high density ligands that induces $2 \rightarrow 1$ transitions. As long as $\Delta J$ is order unity and $\phi_{\text {inc }}$ is not too small for the simulation system size, simulations with other choices of $\Delta J$ and $\phi_{\text {inc }}$ give results that are qualitatively the same as Fig. 4. First of all, typical size of inclusion-rich domains agrees with $L \sim k_{21}^{-1 / 3}$ pretty well for a wide range of $k_{21}$, and the agreement is better when $G$ is smaller [23]. This is because the growth of inclusion-rich domains in the absence of inclusion activities is a twodimensional phase separation dynamics, i.e., $L \sim t^{1 / 3}$ [24, 25, 26]. This growth eventually saturates due to state- 1 to state- 2 transitions on time scale $\sim k_{21}{ }^{-1}$, thus the typical length 
scales of inclusion clusters in the steady state for $G \lesssim \mathcal{O}(1)$ agrees with $L \sim k_{21}{ }^{-1 / 3}[\underline{[5]}$ ]. This relation is also observed in simulations with other $\phi_{\text {inc }}, J_{m n}$, and $k_{12}$ (data not shown). Finite size domains with $1 / 3$ scaling law due to similar mechanisms have been observed in domains in membranes induced by continuous recycling processes [3], and membranes made of binary reactive lipids [7, 8]. Another interesting result is that small inclusion-rich clusters with $M_{\max } \lesssim 10$ are observed when both $k_{21} \Delta t \gtrsim 10^{-3}$ (i.e, $k_{21} \gtrsim 10^{2} \mathrm{~s}^{-1}$ ) and $k_{12} \Delta t \lesssim 10^{-2}$ (i.e., $k_{12} \lesssim 10^{3} \mathrm{~s}^{-1}$ ) are satisfied. Therefore in systems with interaction energy and active transition rates comparable to the characteristic time scale of a motor protein, the lifetime of state-1 inclusions could be too short for a large cluster to be formed. In [14], enhanced clustering of light-sensitive bacteriorhodopsin (BR) is observed in the presence of light, but there are only a few BR in a typical cluster. A possible explanation for this observation is that in the absence of light, BRs have very weak interaction with each other (like our simulation with $k_{12}=0$ and all inclusions are in state-2); while in the presence of light, BRs in an intermediate state (analogous to state-1 inclusions in our simulation) have attractive interaction with each other, so BRs begin to form clusters. However, the lifetime of this intermediate (for BR, it is on the order of $1 \mathrm{~ms}$ ) is too short for large clusters to be formed, thus only very small BR clusters are observed.

Figure 5 shows the relation between $L$ and $k_{12}$ for $\phi_{\text {inc }}=12.5 \%, \Delta J=1.5 k_{B} T$, and $k_{21}=10 \mathrm{~s}^{-1}$ on a $128 \times 128$ lattice. Here $L$ shows a switch-like dependence on $k_{12}: L$ depends weakly on $k_{12}$ when $k_{12}$ is less than some crossover rate $k_{12}^{*}$ but increases rapidly with $k_{12}$ when $k_{12} \gtrsim k_{12}^{*}$. This can be understood by comparing $L_{2} \sim \sqrt{\left(\frac{a^{2}}{\Delta t}\right) k_{12}{ }^{-1}}$, the diffusion length of a state-2 inclusion within its lifetime, to $L$. When $L_{2} \gtrsim L$, state-2 inclusions in inclusion-rich domains can escape to inclusion-poor domains within their lifetime, thus $L$ depends weakly on $k_{12}$. On the other hand, as illustrated in the inset concentric circles of Fig. 5, when $L_{2} \lesssim L$, the chance that a state-2 inclusion in an inclusion-rich domain cannot escape to inclusion-poor domains increases rapidly as $\Delta L=L-L_{2}$ increases. In this case $L$ increases rapidly with $k_{12}$. Thus $k_{12}^{*}$ is determined by the condition $\sqrt{\left(\frac{a^{2}}{\Delta t}\right) k_{12}^{*-1}} \sim L$. As can be seen from Fig. 5, for given $G$, the crossover occurs when $k_{12} \Delta t \sim(L / a)^{-2}$, and the switch-like behavior is more significant for systems with smaller $G$. For simulation with $k_{21}>\mathcal{O}\left(10 \mathrm{~s}^{-1}\right)$, smaller $L$ causes $k_{12}^{*}$ to be larger, and the switch-like behavior is less significant because simulations are performed with $k_{12} \Delta t \leq \mathcal{O}(1)$. In the limit of very large $k_{12}$, one expects that almost no state- 2 inclusion can escape from the domain within 
its lifetime, and typical size of inclusion-rich domains should saturate to its equilibrium value either determined by system size or (when $G$ is large) by the length scale selected by inclusion-membrane coupling. However, this limit happens when $k_{12} \Delta t \geq \mathcal{O}(1)$, and therefore cannot be accurately simulated in the present work.

The inclusion-curvature coupling $G$ also has important effects on $L$. First, both Fig. 4 and Fig. 5 show that $L$ decreases as $G$ increases. That is, the coupling between inclusion density and membrane curvature suppresses the formation of large inclusion clusters because the inclusions tend to locate in regions with greater membrane curvature. As a result, instead of forming large inclusion-rich domains, the membrane prefers to form many mountain-like regions with inclusions aggregating on them when $G \neq 0[4]$. Second, $L \sim k_{21}^{-1 / 3}$ relation in Fig. 4 does not describe large $G$ cases as well as small $G$ cases because besides the length scale set by inclusion transitions, inclusion-curvature coupling provides another length scale for inclusion-rich domains. This is also true in Fig. 5, where the rapid increase of $L$ at $k_{12}>k_{12}^{*} \sim(L / a)^{-2} / \Delta t$ is not significant at large $G$. Thus, in general inclusion-curvature couplings competes with the effects of $k_{\alpha \beta}$.

In summary, our study shows that there are several ways for inclusion activities to control the typical size of nonequilibrium membrane domains. In particular, the power-law relation $L \sim k_{21}{ }^{-1 / 3}$ provides a way to continuously tune the size of domains, the switch-like dependence of $L$ on $k_{12}$ provides a mechanism for sudden change of domain size dependence on the inclusion activities. Although our model has neglected hydrodynamics of the solvent and active forces exerted by the inclusions, the mechanism proposed in the current study is rather general and should exist in models which take these effects into account. A detailed analysis that includes the effects of active forces and full hydrodynamics will be presented in a future work [27]. The small inclusion clusters in the large $k_{21}$ regime observed in our simulations are similar to the experimental observations in [14]. We expect more experiments on these systems and other membranes can be performed to study the mechanisms revealed in our model. For example, the different hydrophobic lengths of different internal states of the rhodopsin [28] can be used to induce effective interaction between nearby rhodopsin, therefore a rhodopsin-containing membrane is also a good candidate for experimental study.

This work is supported by National Science Council of the Republic of China under Grant No. NSC-93-2112-M-008-020. Part of this work is done during HYC's visit to the National Center for Theoretical Sciences (NCTS), Hsinchu, Taiwan. 
[1] H. Lodish et al., Molecular cell biology, 3rd ed. (W.H. Freeman, New York, 1995), 3rd ed.

[2] G. Vereb et al, Proc. Natl. Acad. Sci. USA 100, 8053 (2003); and K. Simons and E. Ikonon, Nature 387, 569 (1997).

[3] M.S. Turner, P. Sens, and N. D. Socci, Phys. Rev. Lett. 95, 168301 (2005); and L. Foret, Europhys. Lett. 71, 508 (2005).

[4] E.J. Wallace, N.M. Hooper, and P.D. Olmsted, Biophys. J. 88, 4072 (2005).

[5] H-Y. Chen, Phys. Rev. Lett. 92, 168101 (2004).

[6] D. Lacoste and A.W.C. Lau, Europhys. Lett. 70, 418 (2005).

[7] R. Reigada, J. Buceta, and K. Lindenberg, Phys, Rev. E 71, 051906 (2005).

[8] R. Reigada, J. Buceta, and K. Lindenberg, Phys, Rev. E 72, 051921 (2005).

[9] M.C. Sabra and O.G. Mouritsen, Biophys. J. 74, 745 (1998).

[10] J. Prost and R.Bruinsma, Europhys. Lett. 33, 321 (1996).

[11] S. Ramaswamy, J. Toner, and J. Prost, Phys. Rev. Lett. 84, 3494 (2000).

[12] J.-B. Manneville, P. Bassereau, S. Ramaswamy, and J. Prost, Phys. Rev. E 64, 021908 (2001).

[13] M. Rao and R.C. Sarasij, Phys. Rev. Lett. 87, 128101 (2001).

[14] N. Kahya, D.A. Wiersma, B. Poolman, and D. Hoekstra, J. Biol. Chem. 277, 39304 (2002).

[15] S. Scheuring and J.N. Sturgis, Science 309, 484 (2004).

[16] This relation can be obtained from a simple microscopic model similar to the one presented in Sec.6.3 of S.A. Safran, Statistical Thermodynamics of Surfaces, Interfaces, and Membranes (Addison-Wesley, Reading, MA, 1994).

[17] R. Goetz, G. Gompper, and R. Lipowsky, Phys. Rev. Lett., 82, 221, (1999).

[18] B. Różycki, R. Lipowsky, and T.R. Weikl, Phys. Rev. Lett., 96, 048101 (2006).

[19] P.F.F. Almeida and W.L.C. Vaz, in Handbook of Biological Physics, Vol 1, edited by R. Lipowsky and E. Sackmann (Elsevier/NorthHolland, Amsterdam, 1995).

[20] For equilibrium segregative instabilities of two-component membranes, see, for example, P. B. Sunil Kumar and M. Rao, Phys. Rev. Lett. 80, 2489 (1998). Our study has different predictions from this reference because we study noneqilibrium systems.

[21] H. Strey, M. Peterson, and E. Sackmann, Biophys. J. 69, 478, (1995).

[22] D. Needham and R. M. Hochmuth, Biophys. J. 61, 1664 (1992). 
[23] The small deviation of $L$ from the $-1 / 3$ power law in the small $G$, small $k_{21} \Delta t$ region is due to finite size effect because typical number of inclusions in a cluster in our simulation becomes comparable to the total number of inclusions in this region.

[24] M. Seul, N.Y. Morgan, and C. Sire, Phys. Rev. Lett. 73, 2284 (1994).

[25] A. J. Bray, Adv. Phys. 43, 357, (1994).

[26] P.B. Sunil Kumar, G. Gompper, and R. Lipowsky, Phys. Rev. Lett. 86, 3911 (2001).

[27] H-Y. Chen and C-H. Chen, unpublished.

[28] J. Isele, T.P. Sakmar, and F. Siebert, Biophys. J. 79, 3063 (2000). 


\section{Figure captions}

- FIG. 1: Schematics of a membrane with two-state inclusions.

- FIG. 2: Domain size distribution $P(M)$ for $\phi_{\text {inc }}=12.5 \%, \Delta J=1.5 k_{B} T, k_{12}=$ $10^{2} \mathrm{~s}^{-1}, k_{21}=k_{12} / 32$, and $G=0,1$, and 2 . The peak at $M=1$ comes from the isolated inclusions in the inclusion-poor domain. The peak at greater $M$ provides the characteristic size of inclusion-rich domains. As $G$ increases, the characteristic size of inclusion-rich domains decreases and the peak of $P(M)$ becomes more significant due to inclusion-membrane coupling.

- FIG. 3: Snapshots for steady state inclusion distribution (left, dark regions are occupied by inclusions) and membrane height (right) for $\phi_{\mathrm{inc}}=12.5 \%, \Delta J=1.5 k_{B} T$, $k_{12}=10^{2} \mathrm{~s}^{-1}$, and $k_{21}=k_{12} / 32$. (a). $G=0,(\mathrm{~b}) . G=2$. In order to faithfully present the morphology of the membrane, the unit length for $h$ and the unit length in the $x y$ plane are both chosen to be $a$. For nonzero $G$ membrane curvature close to the inclusion-rich domains increases and the typical size of inclusion-rich domain decreases.

- FIG. 4: Typical size of inclusion-rich domains in the steady state for $\phi_{\mathrm{inc}}=12.5 \%$, $\Delta J=1.5 k_{B} T, k_{12} \Delta t=10^{-3} . G=0$ (circles), $G=0.5$ (squares), $G=1.0$ (diamonds), and $G=2.0$ (triangles). The dashed line has slope $-1 / 3$.

- FIG.5: Typical size of inclusion-rich domains in the steady state for $\phi_{\text {inc }}=12.5 \%$, $\Delta J=1.5 k_{B} T, k_{21} \Delta t=10^{-4} . G=0$ (circles), $G=0.5$ (squares), $G=1.0$ (diamonds), and $G=2.0$ (triangles). Data with greatest value of $k_{12} \Delta t$ are obtained from simulations on a $256 \times 256$ lattice. The inset is a schematic of a domain with radius $L$. When $k_{12}>k_{12}^{*}$, state-2 inclusions in the inner circle (radius $\Delta L$ ) have small chance to leave the domain within its lifetime. $\Delta L=L-L_{2}$ increases as $k_{12}$ increases. 

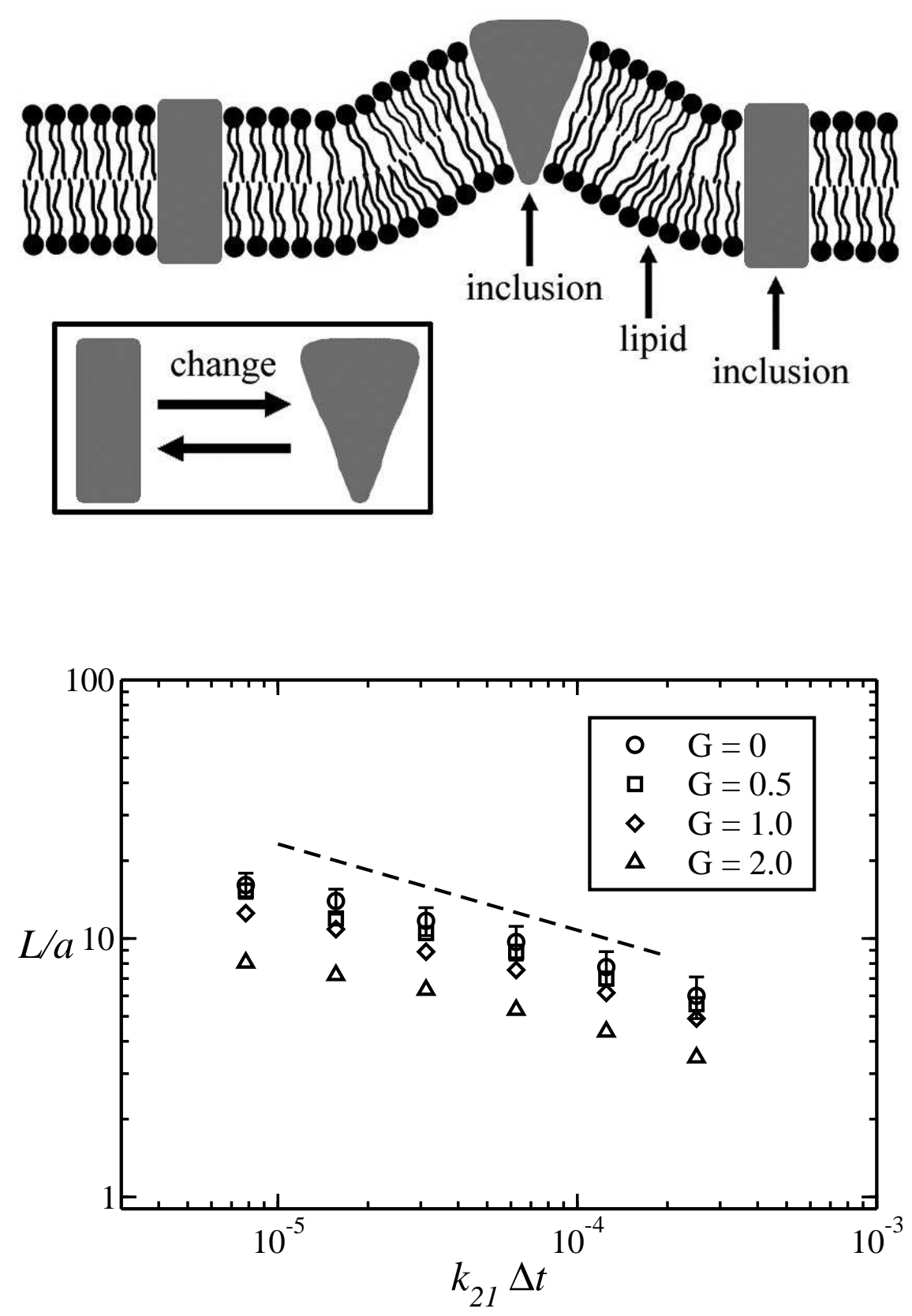

FIG. 2: Domain size distribution $P(M)$ for $\phi_{\text {inc }}=12.5 \%, \Delta J=1.5 k_{B} T, k_{12}=10^{2} \mathrm{~s}^{-1}, k_{21}=$ $k_{12} / 32$, and $G=0,1$, and 2 . The peak at $M=1$ comes from the isolated inclusions in the inclusion-poor domain. The peak at greater $M$ provides the characteristic size of inclusion-rich domains. As $G$ increases, the characteristic size of inclusion-rich domains decreases and the peak of $P(M)$ becomes more significant due to inclusion-membrane coupling. 

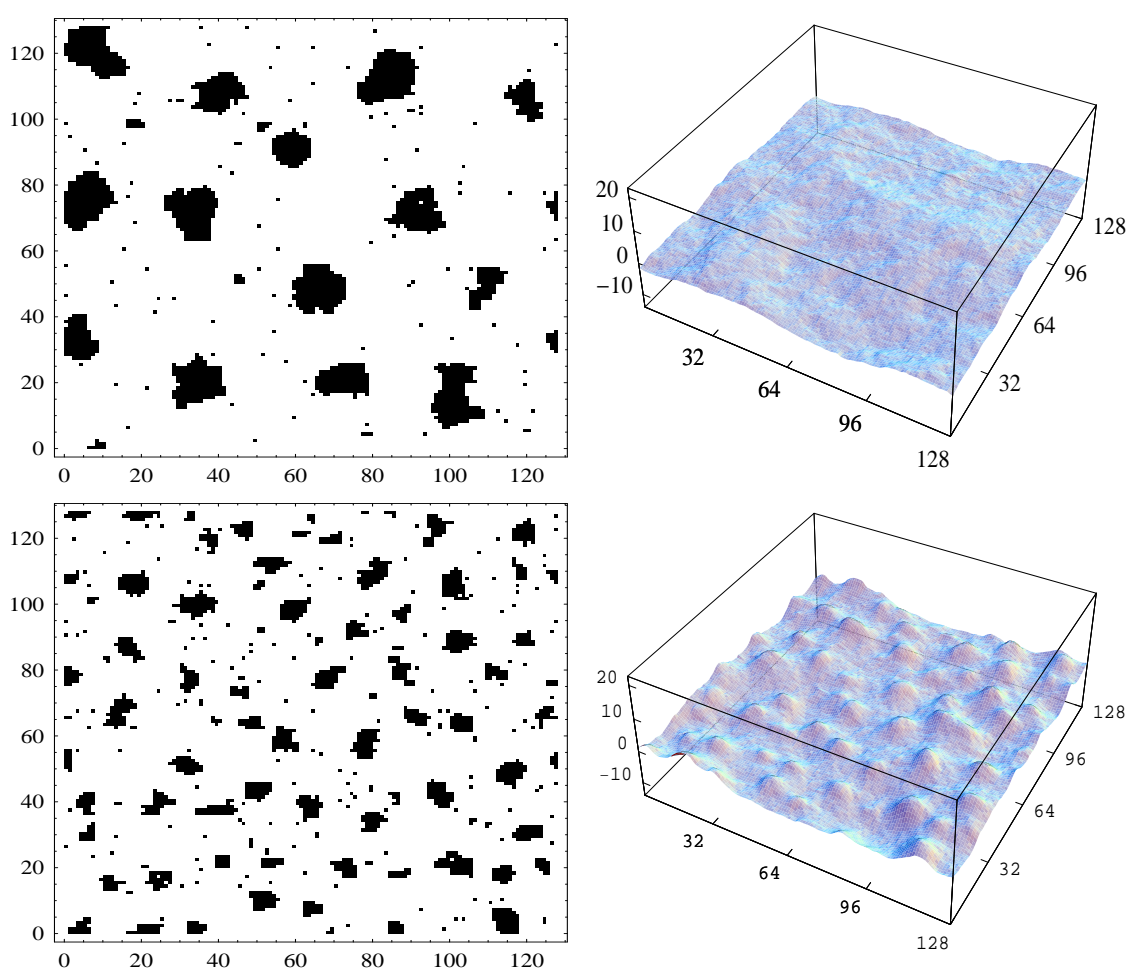

FIG. 3: Snapshots for steady state inclusion distribution (left, dark regions are occupied by inclusions) and membrane height (right) for $\phi_{\text {inc }}=12.5 \%, \Delta J=1.5 k_{B} T, k_{12}=10^{2} \mathrm{~s}^{-1}$, and $k_{21}=k_{12} / 32$. (a). $G=0,(\mathrm{~b}) . \quad G=2$. In order to faithfully present the morphology of the membrane, the unit length for $h$ and the unit length in the $x y$ plane are both chosen to be $a$. For nonzero $G$ membrane curvature close to the inclusion-rich domains increases and the typical size of inclusion-rich domain decreases. 


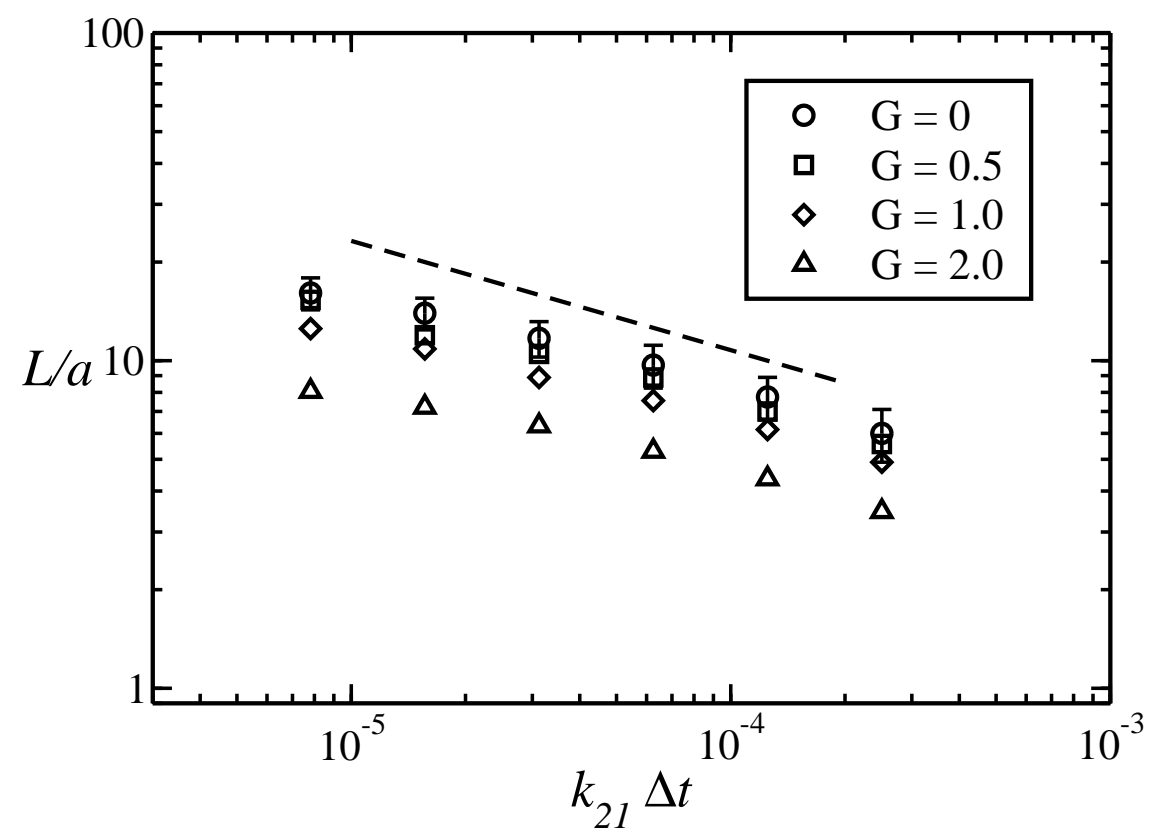

FIG. 4: Typical size of inclusion-rich domains in the steady state for $\phi_{\text {inc }}=12.5 \%, \Delta J=1.5 k_{B} T$, $k_{12} \Delta t=10^{-3} . G=0$ (circles), $G=0.5$ (squares), $G=1.0$ (diamonds), and $G=2.0$ (triangles). The dashed line has slope $-1 / 3$. 


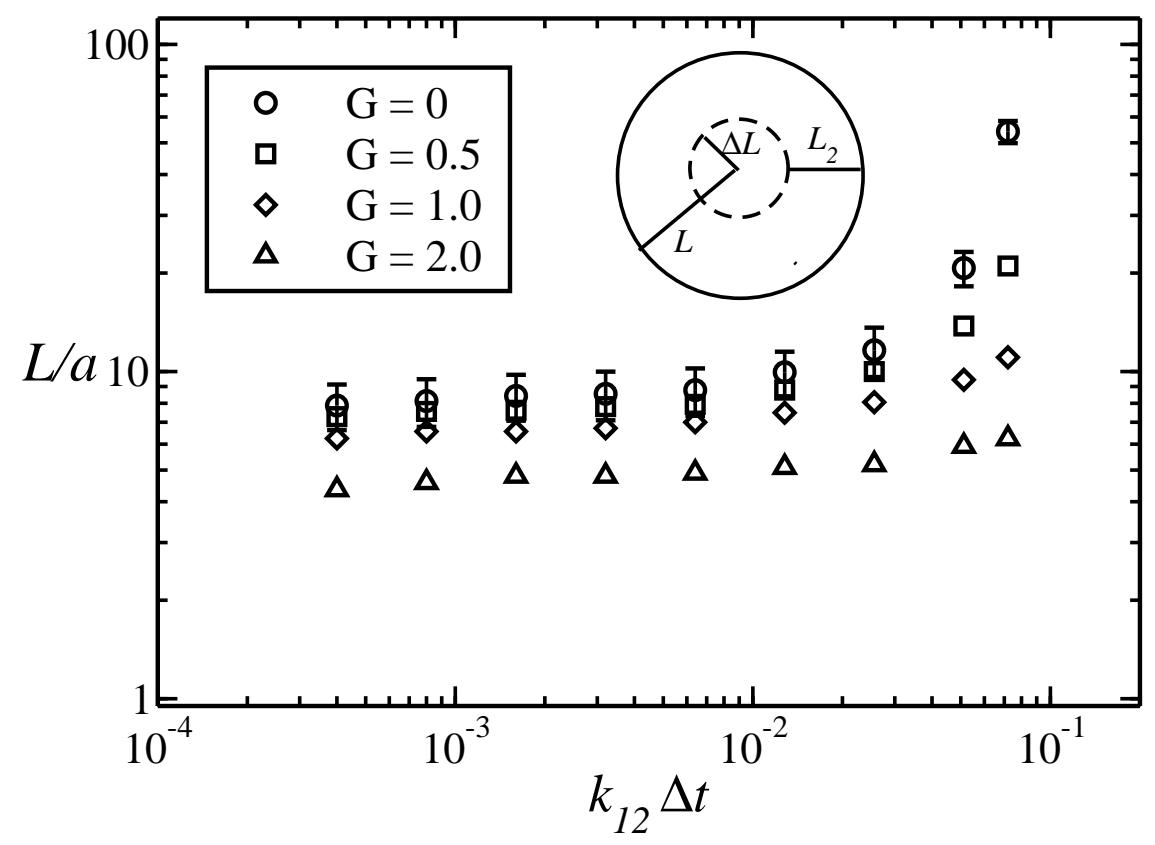

FIG. 5: Typical size of inclusion-rich domains in the steady state for $\phi_{\text {inc }}=12.5 \%, \Delta J=1.5 k_{B} T$, $k_{21} \Delta t=10^{-4} . G=0$ (circles), $G=0.5$ (squares), $G=1.0$ (diamonds), and $G=2.0$ (triangles). Data with greatest value of $k_{12} \Delta t$ are obtained from simulations on a $256 \times 256$ lattice. The inset is a schematic of a domain with radius $L$. When $k_{12}>k_{12}^{*}$, state-2 inclusions in the inner circle (radius $\Delta L$ ) have small chance to leave the domain within its lifetime. $\Delta L=L-L_{2}$ increases as $k_{12}$ increases. 